\title{
Successful pregnancy after conservative management of early stage endometrial carcinoma in a young nulliparous woman
}

\author{
Magdalena Magnowska ${ }^{1}$, Ewa Nowak-Markwitz ${ }^{1}$, Andrzej Frankowski², Wojciech Rzeszutko \\ Marek Spaczyński ${ }^{1}$
}

\begin{abstract}
${ }^{1}$ Department of Gynecologic Oncology, Chair of Gynecology, Obstetrics and Gynecologic Oncology, Poznan University of Medical Sciences, Poznan, Poland ${ }^{2}$ Department of Biochemistry and Pathomorphology, Chair of Gynecology, Obstetrics and Gynecologic Oncology, Poznan University of Medical Sciences, Poznan, Poland ${ }^{3}$ Department of Pathological Anatomy, Silesian Piasts University of Medicine, Wroclaw, Poland
\end{abstract}

Submitted: 27 September 2012

Accepted: 25 December 2012

Arch Med Sci 2015; 11, 1: 237-239

DOI: 10.5114 /aoms.2013.36183

Copyright (c) 2015 Termedia \& Banach

The incidence of endometrial cancer (EC) in the population of young women has been increasing. Total hysterectomy with bilateral salpingo-oophorectomy remains the standard therapy for early endometrial cancer [1]. However, doctor and patient attitudes towards radical cancer treatment methods, especially in young women of reproductive age, have been noticeably changing [1-4]. Women expect doctors to apply fertility-sparing therapy, if possible. Early endometrial cancer cases treated with preservation of reproductive function methods have been reported [5].

We present a case of a patient who conceived spontaneously and delivered a healthy newborn after hormonal treatment of early endometrial cancer.

A 29-year-old female patient, nullipara, obese (body mass index (BMI) $32.1 \mathrm{~kg} / \mathrm{m}^{2}$ ), presented to her gynecologist with a complaint of irregular, heavy bleeding. For the last year the menstrual pattern was irregular, every 30-60 days, hypermenorrhea lasting up to 14 days. An ultrasound examination revealed endometrial hypertrophy and polyp suspicion. The patient underwent diagnostic dilation and curettage. Histopathology revealed endometrial hyperplasia with atypia with adenocarcinoma foci (Figure 1). Magnetic resonance imaging and chest $\mathrm{X}$-ray excluded more advanced cancer. Treatment options and the risk of cancer progression in case of saving childbearing potential were discussed with the patient, who eventually chose fertility-sparing therapy. She was strongly advised to lose weight with the help of a professional dietician. The patient was treated with high-dose oral progesterone, megestrol acetate $160 \mathrm{mg} /$ day for 6 months after inserting the levonorgestrel intrauterine system (LNG-IUD). Endometrial curettage was performed after removing the levonorgestrel intrauterine system after 6 months. The histopathological examination revealed endometrium with decidual transformation. Mostly the endometrial glands were inactive. Single glands showed signs of hyperplasia without direct atypia. The assessment of atypia was difficult because of the gestagen influence (Figure 2). By that time she had reduced her weight by $30 \mathrm{~kg}\left(\mathrm{BMI} 22 \mathrm{~kg} / \mathrm{m}^{2}\right)$. Then she was given estro-
Corresponding author:

Magdalena Magnowska MD, PhD

Department of Gynecologic

Oncology

Chair of Gynecology,

Obstetrics and Gynecologic

Oncology

Poznan University

of Medical Sciences

33 Polna St

60-535 Poznan, Poland

Phone: +48 618419330

Fax: +48 616599645

E-mail:magna5@op.pl 


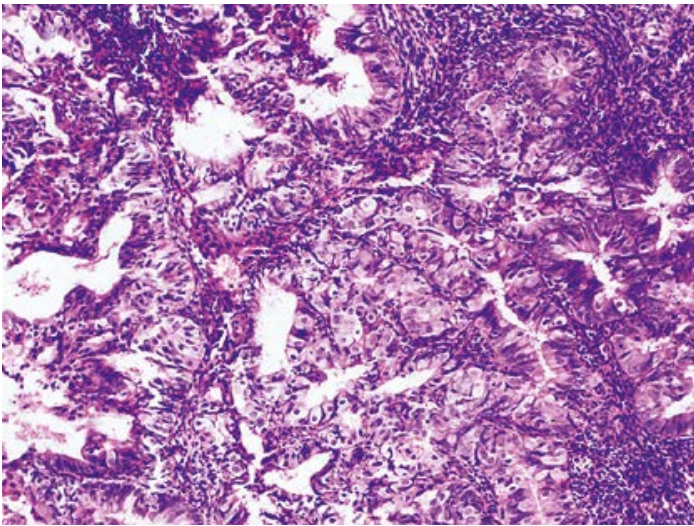

Figure 1. Pre-therapy. Endometrial glands with neoplastic transformation $(H+E, 100 x)$

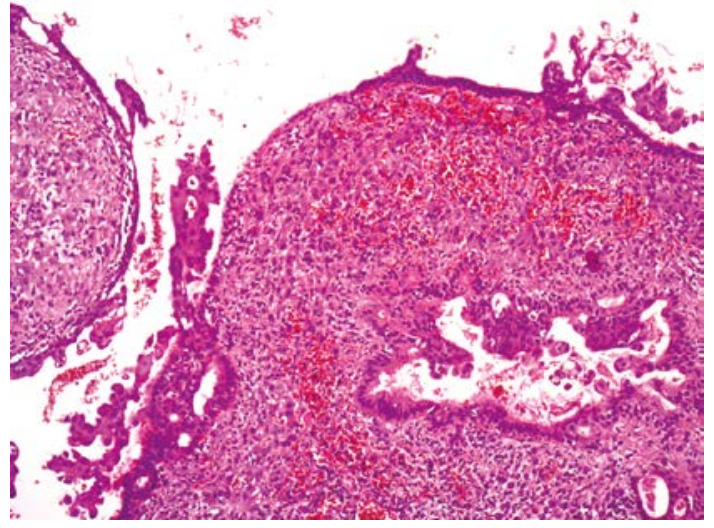

Figure 2. Post-therapy with high-dose systemic progesterone and local LNG-IUD for 6 months. Stroma with decidual transformation (drug-induced). Hyperplasia within the glandular tubes and on the endometrium surface - foci of residual hyperplasia $(\mathrm{H}+\mathrm{E}, 50 \mathrm{x})$

ian tumors or metastases, no suspected lymph nodes, and no contraindications for endocrine therapy. Aggressive tumors such as papillary serous, clear cell adenocarcinoma, adenosquamous carcinoma and carcinosarcomas are contraindications for conservative management, and patients with these tumor types should be discouraged from attempting conservative treatment with hormones [6].

The current FIGO classification (2009) for endometrial cancer divides stage I into IA and IB, depending on the depth of myometrial invasion (MI). It is not always possible to evaluate it in the material from dilation and curettage (D\&C). The best method of assessing the myometrial invasion and extrauterine spread before surgery is contrast-enhanced magnetic resonance imaging (MRI) [7]. The $D \& C$ is preferred to endometrial biopsy and seems to comply with the need for thorough evaluation of grade and occult malignancy [6]. Evaluation of the endometrial tissue should determine the lymphovascular space invasion (LVSI), which is considered a risk factor for lymph node metastasis. A literature search revealed that in case of LVSI the incidence of pelvic lymph node involvement grows from $7 \%$ to $27 \%$ and the 5 -year survival rate decreases from $86 \%$ to only $61 \%$ of patients [8].

The first-line hormonal treatment for early endometrial cancer is medroxyprogesterone acetate (MPA) (dosage 200-800 mg/day) or megestrol acetate (MA) (10-400 mg/day), which inhibit the estrogen receptor and endometrial cell mitosis, promote apoptosis and induce secretory endometrium [9]. Mostly, 6 months of continuous therapy is enough to obtain remission, although there are cases with a 9-month hormonal treatment [10]. Our patient also did not respond completely after 6 months, despite the fact that she received combined therapy: megestrol acetate and the LNG-IUD. 
We obtained the resolution of adenocarcinoma foci, but the residual foci of atypical hyperplasia were still present. Although it seemed risky, with patient consent and wish and after thorough in formation, the chosen strategy was to conceive as soon as possible. Only one similar situation has been published. There is a report from a prospec tive study in which women were encouraged to conceive before the complete response to hormonal treatment. Remission was obtained by means of a low-dose cyclic natural progestin therapy (200 mg/day from day 14-25). The response rate was $57 \%$ and $43 \%$ of women conceived, of which 8 were in persistent disease or partial response. Another 3 complete responses were obtained after the delivery [11].

In conclusion, the case illustrates the favorable outcome of pregnancy after high-dose systemic and local progesterone therapy in early stage focal adenomatous endometrial carcinoma with atypical hyperplasia. Six months of continuous therapy and 6 months of cyclical progesterone therapy may achieve a good endometrial response for resolution of focal endometrial carcinoma, and this may favor implantation after the risk factors such as obesity have been controlled.

\section{Conflict of interest}

The authors declare no conflict of interest.

\section{References}

1. Gottwald L, Pluta P, Piekarski J, et al. Long-term survival of endometrioid endometrial cancer patients. Arch Med Sci 2010; 6: 937-44.

2. Maltaris T, Boehm D, Dittrich R, et al. Reproduction beyond cancer: a message of hope for young women. Gynecol Oncol 2006; 103: 1109-21.

3. Sikora-Szczęśniak D, Sikora W. Fertility sparing surgical treatment of malignant ovarian tumors in the reproductive age group of women. Ginekol Pol 2012; 83: 27-32.

4. Markowska A, Pawłowska M, Markowska J, et al. Influence of oncological treatment on fertility of women and pregnancy outcome. Ginekol Pol 2010; 81: 446-51.

5. Olejek A, Olszak-Wąsik K, Horzelski T, et al. Endometrial cancer in a 25 -year-old patient. Case study. Ginekol Pol 2012; 83: 224-8.

6. Tangjitgamol S, Manusirivithaya S, Hanprasertpong J. Fertility-sparing in endometrial cancer. Gynecol Obstet Invest 2009; 67: 250-68.

7. DelMaschio A, Vanzulli A, Sironi S, et al. Estimating the depth of myometrial involvement by endometrial carcinoma: efficacy of transvaginal sonography vs. MR imaging. AJR Am J Roentgenol 1993; 161: 595-9.

8. Zaino R, Kurman R, Diana K, Morrow C. Pathologic models to predict outcome for women with endometrial adenocarcinoma: the importance of the distinction between surgical and clinical stage - a Gynecologic Oncology Group study. Cancer 1996; 77: 1115-21.

9. Sacgusa M, Okayasu I. Progesterone therapy for endometrial carcinoma reduces cell proliferation but does not alter apoptosis. Cancer 1998; 83: 111-21.
10. Kaku T, Yoshikawa H, Truda H, et al. Conservative therapy for adenocarcinoma and atypical endometrial hyperplasia of the endometrium in young women: central pathologic review and treatment outcome. Cancer Lett 2001; 167: 39-48.

11. Signorelli M, Caspani G, Bonazzi C, et al. Fertility-sparing treatment in young women with endometrial cancer or atypical complex hyperplasia: a prospective single-institution experience of 21 cases. BJOG 2009; 116: 114-8. 